10.1590/S1414-40772018000300007

Este é um artigo publicado em acesso aberto sob uma licença Creative Commons

https://creativecommons.org/licenses/by-nc/4.0/

\title{
Formação profissional para o SUS: análise de reformas curriculares em cursos de graduação em nutrição
}

\author{
Elisabetta Recine ${ }^{1}$ \\ Kelly Poliany de Souza Alves ${ }^{2}$ \\ Estelamaris Monego ${ }^{3}$ \\ Andrea Sugai ${ }^{4}$ \\ Alice Cristina Medeiros Melo ${ }^{5}$
}

Resumo: A reorganização do modelo assistencial do Sistema Único de Saúde (SUS) se constitui em um desafio e a formação dos profissionais que o integram se apresenta como uma das barreiras para implementação da atenção integral à saúde. Dessa maneira, o objetivo desse estudo foi relatar e problematizar experiências de mudanças na formação de nutricionistas em instituições de ensino superior/IES públicas e privadas, de diferentes regiões do país, que adotaram a formação para o SUS como eixo norteador. Foram avaliados nove cursos, cujos resultados foram sintetizados em quatro eixos: integração com serviços de atenção à saúde; atuação prática; abordagem integrada do conhecimento e; articulação da formação com projetos de pesquisa e extensão. Estratégias indutoras de reestruturação da formação profissional, como PET-Saúde e Pró-Saúde, contribuíram na interlocução das IES com gestores e profissionais de saúde e na inserção oportuna dos estudantes na rede de serviços. Reformulações pedagógicas amplas nas IES oportunizaram mudanças mais inovadoras, estruturais e sustentadas. No entanto, permanecem desafios como articulação interna dos professores de diferentes áreas de conhecimento, formação pedagógica dos docentes e inclusão de gestores e profissionais do SUS no planejamento do processo de ensino-aprendizagem em serviço.

Palavras-chave: Formação profissional. Nutricionista. Sistema Único de Saúde.

\section{Workforce development for the brazilian National Health System (SUS): analysis of curricular reforms in nutrition undergraduate courses}

Abstract: The reorganization of the assistance model of the Brazilian National Health System (SUS) is a permanent challenge and the adequate professional training is one of the hindrances for a comprehensive health care. The goal of this study is to analyze the experiences of curricular reform in undergraduate courses in Nutrition in public and private Universities, which have adopted the necessities of the SUS as a main drive for the professional training. Nine undergraduate courses were analyzed and the results were organized in four action axes, i.e.: cooperation with health services; praxis; integral approach to knowledge and; articulation with academic research and extension projects. Government inductor strategies have been supporting the interlocution among the academic institutions and health services and have been facilitating an adequate insertion of students in health services. Broad pedagogical reformulations mainly in public universities gave rise to structural changes. Nevertheless, some difficulties remain, such as the articulation of the academic staff with others health areas at the same institution; pedagogical training programs for the academic staff; participation of health professionals on the planning of teaching learning process.

Key words: Capacity Building. Nutritionist. Brazilian Health National System. 


\section{Introdução}

Dentre as mudanças e os desafios decorrentes do processo de construção e aprimoramento do Sistema Único de Saúde (SUS), é prioritária a organização de um novo modelo assistencial frente às críticas ao modelo biomédico hegemônico que, pela abordagem mecanicista e fragmentada do corpo, dá ênfase a medicina curativa, tecnicista e especializada, que prioriza condições agudas, apresentando insuficiente resolubilidade diante de uma situação epidemiológica onde predominam os quadros crônicos (MENDES, 2011). Apesar das críticas a esse modelo, observa-se que suas principais características continuam presentes na formação e na prática dos diferentes profissionais de saúde e constituem-se como uma das barreiras para a implementação da atenção integral à saúde (GIL et al., 2008).

Para implementação de um novo modelo assistencial, o 'trabalho em equipe multiprofissional', a 'atuação interdisciplinar' e o 'cuidado integral' têm sido características cada vez mais necessárias na atenção à saúde e demandam competências correspondentes pelos profissionais que atuam no SUS. Esse contexto desafia e demanda ajustes das instituições de ensino superior (IES) para elegerem estratégias de renovação, coerentes com a construção e o aprimoramento do SUS (BRASIL, 2016b; CECCIM; FEURWERKER, 2004).

As Diretrizes Curriculares Nacionais (DCN) dos cursos de graduação na área da saúde, publicadas entre 2001 e 2002, preveem, em sua maioria, que a formação do profissional de saúde deve contemplar o sistema de saúde vigente no país, o trabalho em equipe e a atenção integral à saúde (ALMEIDA, 2003). Porém, Ceccim e Feuerwerker (2004) destacam que as DCN se constituem como uma indicação ou uma recomendação, já que, no Brasil, as universidades possuem autonomia, apontada na Lei de Diretrizes e Bases da Educação Nacional (LDB) como uma "[...] prerrogativa de criação, expansão, modificação e extinção de cursos e programas de educação superior; fixação dos currículos de seus cursos e programas; planificação e programação de pesquisa científica e de atividades de extensão, além da elaboração da programação dos cursos" (BRASIL, 2016b, p. 1402). Ceccim e Feurwerker (2004) também ressaltam que novos passos, para além das DCN, são necessários para que as mudanças na formação ocorram amplamente nos cursos de graduação em saúde.

Somando-se à proposta das DCN, alguns programas compartilhados entre os Ministérios da Saúde e da Educação vêm promovendo a aproximação entre o ensino e os serviços de saúde, com vistas a qualificar a formação de profissionais para a atuação no SUS (BRASIL, 2005; 2007b; 2010). O Programa Nacional de Reorientação da Formação Profissional em Saúde (Pró-Saúde) (BRASIL, 2003; 2005) e o Programa de Educação pelo 
Trabalho para a Saúde (PET-Saúde) (BREHMER; RAMOS, 2014), criados em 2005 e 2010, respectivamente, apostaram na integração ensino-serviço que visa a inserção dos estudantes no cenário real de práticas do SUS, com ênfase na atenção básica, desde o início de sua formação, como mecanismo fundamental para transformar o aprendizado, com base na realidade socioeconômica e sanitária da população brasileira e nas demandas concretas dos serviços de saúde.

Apesar de potencialmente benéfica para as instituições formadoras e serviços de saúde, a relação existente entre ambos é complexa, marcada por desafios para o estabelecimento de diálogo e de pactuação entre os envolvidos, o que limita as possibilidades de construção conjunta de mudanças na concepção e nas práticas de cuidado em saúde e a melhor formação profissional (ALBUQUERQUE et al., 2008). Novos perfis delineados e necessários para os profissionais de saúde demandam das IES a adoção de propostas pedagógicas que incluam o emprego de metodologias de ensino condizentes com os resultados esperados. Nesse sentido, o uso de metodologias ativas tem crescido nos cursos de graduação em saúde (BERBEL, 2011). Trata-se de uma concepção educativa que estimula os processos de ensino-aprendizagem crítico-reflexivos que utilizam a problematização como estratégia principal para o envolvimento ativo dos estudantes em seu próprio processo de formação e o vínculo precoce com a realidade e a prática (MITRE et al., 2008; SOBRAL; CAMPOS, 2012).

A educação interprofissional também tem sido experimentada na formação de profissionais de saúde, partindo do pressuposto de que para fazer junto no cotidiano do cuidado em saúde é preciso aprender junto sobre o trabalho em saúde (BATISTA; GERAB; BATISTA, 2013). Mas, a efetivação dessa proposta requer mais do que simplesmente reunir estudantes de diferentes cursos em um ambiente. Para Aguilar-da-Silva, Scapin e Batista (2011, p. 170) é preciso desenvolver recursos que possibilitem "entender como as múltiplas dimensões de qualquer problema de saúde e a sua abordagem por distintas profissões se apresentam e se articulam na determinação do processo saúde-doença".

Nesse contexto, as mudanças nos cursos de graduação em nutrição, com vistas à formação de um profissional qualificado para a atuação no SUS, têm se apresentado muito mais complexas do que apenas a inclusão de novas disciplinas ou de novos conteúdos em modelos curriculares distintos. A atual organização do SUS e a perspectiva do alcance da Atenção Nutricional expressa na Política Nacional de Alimentação e Nutrição (PNAN) exigem o desenvolvimento de competências que permitam ao nutricionista atuar em uma rede de atenção à saúde composta por serviços de diferentes densidades tecnológicas, em interação 
com diferentes profissionais e usuários, onde os conhecimentos e as práticas relativas às diversas áreas que compõe o campo de alimentação e nutrição possam ser acionados e integrados, de acordo com as demandas e as necessidades de saúde, identificadas no lócus de inserção do profissional (BRASIL, 2012).

Segundo os dados do Cadastro Nacional de Estabelecimentos de Saúde (CNES), em maio de 2016, aproximadamente 18,8 mil nutricionistas estavam cadastrados como profissionais atuantes em estabelecimentos de saúde que prestavam serviços ao SUS (BRASIL, 2016a). Desses, 33\% vinculados aos serviços responsáveis pela oferta de Atenção Básica à saúde (Unidades Básicas de Saúde, Centros Municipais de Saúde, Núcleos de Apoio à Saúde da Família, Academias da Saúde), 50\% em serviços de Atenção Especializada Hospitalar (Hospitais gerais e especializados), 13\% em serviços de Atenção Especializada Ambulatorial (Centros de Especialidades, Policlínicas, Centros de Atenção Psicossocial, Serviços de Atenção Domiciliar) e 4\% vinculados a outros lócus do SUS (Gestão, Vigilância em Saúde, Farmácias, Laboratórios, Centrais de Regulação).

Diante desse cenário, o presente artigo tem por objetivo relatar e problematizar experiências de mudanças curriculares para a formação de nutricionistas em cursos de graduação em instituições públicas e privadas, de diferentes regiões do país, que adotaram a formação para o SUS como eixo norteador.

\section{Caminhos do estudo}

Nessa pesquisa, pretendeu-se compreender a formação profissional em nutrição para o SUS, a partir da perspectiva sócio-histórica no campo da saúde e da educação e, sobretudo, por meio dos documentos e relatos de reestruturação curricular das IES selecionadas. A sistematização do levantamento bibliográfico e documental apoiou-se em registros oficiais e artigos que reorientam a formação profissional em saúde no Brasil e a integração ensinoserviço, destacando-se as metodologias inspiradas em pressupostos pedagógicos não diretivos, difundidas pelo Ministério da Saúde, no campo da formação em saúde, bem como aos princípios e diretrizes do SUS, como a integralidade do cuidado, a interdisciplinaridade e o trabalho em equipe (CONTERNO; LOPES, 2016; MEDEIROS; BRAGA-CAMPOS, MOREIRA, 2014). No campo da nutrição, estabeleceu-se o balizamento teórico considerando o alcance da Atenção Nutricional expressa na PNAN e na garantia da segurança alimentar e nutricional (BRASIL, 2012). 
O cenário onde a situação alimentar e nutricional da população brasileira faz uma interface para a garantia da segurança alimentar e nutricional gera demandas para a ordenação da formação dos profissionais da saúde, sugerindo caminhos que convergem para a agenda da alimentação e nutrição no SUS. Assim, é estratégico considerar não apenas o processo de trabalho em nutrição, saúde e educação como eixos estruturantes para a organização da formação da força de trabalho (BRASIL, 2012), mas as necessidades de saúde, com ênfase nas demandas da população e do SUS, como também de conteúdos que permitam ao profissional atuar nas diferentes fases do sistema alimentar, ampliando sua perspectiva de ação para os determinantes sociais da saúde (RECINE et al., 2012).

Apesar do reconhecimento da importância do tema nas avaliações dos cursos e nos debates sobre a formação do profissional, essa área do conhecimento ainda não alcançou o mesmo estatuto dos conhecimentos biológicos na maioria dos projetos pedagógicos quando da aprovação das DCN (BRASIL, 2001a). Uma tentativa foi a elaboração do Consenso sobre habilidades e competências do nutricionista no âmbito da saúde coletiva, cujo objetivo foi o de caracterizar quais conhecimentos, habilidades, atitudes e formas de pensar são necessárias para as ações profissionais/funções do nutricionista no campo da saúde coletiva (RECINE; SUGAI, 2013).

Nesse contexto, uma estratégia importante seria a articulação dos gestores com as instituições formadoras para o desenvolvimento de projetos de formação em serviço, campos para extensão e pesquisa na rede de atenção à saúde do SUS, possibilitando o desenvolvimento de práticas do cuidado relacionadas à alimentação e nutrição (BRASIL, 2012).

Esse cenário propiciou a demanda de um estudo sobre o papel do profissional nutricionista, incluindo questionamentos e reflexões sobre mudanças e diretrizes curriculares, carga horária e ajustes na formação para responder às demandas da realidade nacional. Sua origem se dá com o projeto matriz "Registro de Experiências de Formação do Nutricionista para o SUS", aprovado pela Comissão de Ética em Pesquisa da Faculdade de Ciências da Saúde da Universidade de Brasília (CEP/FS/UnB), sob protocolo (041745/2015).

Assim, essa pesquisa, de natureza qualitativa, analisou experiências de cursos de graduação em nutrição que realizaram reforma curricular, ou aqueles que já se estabeleceram, tendo como eixo norteador a formação de profissionais para o SUS, buscando reconhecer o processo adotado, a proposta resultante e os resultados disponíveis.

Duas estratégias combinadas e concomitantes foram utilizadas para a captação dos cursos com perfil desejado para o estudo. A primeira foi identificá-los dentre aqueles que 
apresentaram suas experiências no Encontro Nacional de Formação Profissional, promovido pelo Conselho Federal de Nutricionistas (CFN), em setembro de 2013. Em meio as IES participantes, identificaram-se quatro aptas a participar do estudo, que foram convidadas a enviar relatos sobre suas estratégias curriculares, por meio do preenchimento de um formulário eletrônico padronizado.

A segunda estratégia foi a divulgação de uma chamada na Rede de Alimentação e Nutrição do SUS - RedeNutri, replicada nos sítios eletrônicos de entidades profissionais de nutricionistas, convidando as IES a enviar relatos de experiências de reforma curricular realizadas a partir do ano de 2005 (ano de lançamento do Pró-Saúde), a partir do mesmo formulário eletrônico padronizado utilizado na primeira estratégia. Solicitou-se que os relatos fossem preenchidos pelos coordenadores de curso ou docentes que compunham o Núcleo Docente Estruturante (NDE).

O formulário eletrônico continha campos distintos para: identificação da IES, caracterização geral do curso e da proposta curricular atual e; para informações gerais sobre a experiência curricular, sendo ainda possível anexar o Projeto Político Pedagógico (PPP), bem como o Relatório de implementação do currículo e outros arquivos que a instituição julgasse pertinentes.

Foram recebidos dezoito relatos de experiências. Desses, nove IES foram escolhidas para participarem da oficina de trabalho "Experiências de Formação do Nutricionista para o SUS", diante dos seguintes critérios: diversidade quanto à natureza da instituição (pública/privada), diversidade regional, caráter inovador, existência de processos participativos entre os docentes e os discentes na construção da experiência, bem como entre a instituição de ensino, a gestão local do SUS e os profissionais atuantes nos serviços de saúde na construção da experiência e, a sustentabilidade das mudanças empreendidas no curso.

A oficina de trabalho ocorreu nos dias 2 e 3 de julho de 2015, na Universidade de Brasília, com o objetivo de aprofundar o conhecimento acerca das nove experiências selecionadas. Participaram do evento, um total de 26 pessoas, com representantes de cursos das regiões Norte (1 privado), Nordeste (1 privado e 1 público), Centro-oeste (2 públicos), Sudeste (1 privado e 1 público) e Sul (1 privado e 1 público), totalizando quatro cursos de instituições privadas e cinco de instituições públicas. Mediante o consentimento dos participantes, a atividade foi integralmente gravada e transcrita, preservando-se assim, os contextos nos quais os discursos foram produzidos.

Além dos formulários eletrônicos e das transcrições da oficina, foram utilizados como fonte para coleta dos dados, documentos técnicos sobre os cursos, disponíveis nos sítios 
eletrônicos institucionais ou encaminhados pelos representantes dos cursos. A partir dessas fontes, foram organizadas informações sobre os aspectos gerais dos cursos (ano de início das atividades, duração, carga horária, grade de disciplinas, reformas curriculares realizadas, integração com outros cursos, dentre outras) e aspectos específicos sobre as estratégias utilizadas para aprimorar a formação dos nutricionistas para o SUS.

As informações foram analisadas a partir de quatro eixos prioritários definidos a priori e embasados no arcabouço teórico que alicerçou a pesquisa e tem orientado as propostas de atualização da formação profissional em saúde e integração ensino-serviço: a integração com os serviços de atenção à saúde; a atuação prática nos serviços de saúde; a abordagem integrada do conhecimento e; a articulação da formação na graduação com projetos de pesquisa e extensão. $\mathrm{Na}$ apresentação dos resultados dessa análise, os eixos foram agrupados em dois grandes grupos: a integração com os serviços de saúde, onde foram incluídas práticas em serviços e projetos de pesquisa e extensão, e a abordagem integrada do conhecimento.

\section{Aproximando a lente da formação de nutricionistas}

No Brasil, entre as décadas de 1930 a 1960, foram criados sete cursos de nutrição; chegando até 1996 a um total de 45 cursos em todo o país. Após a promulgação da LDB em 1996 (BRASIL, 2016b), que impulsionou o ensino privado, observou-se um crescimento acelerado de cursos, chegando a 169 em 2003 (CALADO, 2003; CANESQUI; GARCIA, 2005) e, atualmente, a mais de 550 cursos (BRASIL, 2017). .

A formulação da LDB teve o objetivo de reestruturar o sistema escolar brasileiro, incluindo os cursos de graduação em saúde, promovendo a extinção dos currículos mínimos e a adoção de Diretrizes Curriculares Nacionais (DCN), específicas para cada curso superior (BRASIL, 2016b). As DCN para os cursos de nutrição foram publicadas em 2001 e definiram um novo perfil para o profissional nutricionista (BRASIL, 2001b). Desde então, diversos cursos têm buscado adaptar seus currículos para formar profissionais que estejam em sintonia com o perfil definido:

[...] O profissional nutricionista deve ter uma formação generalista, humanista e crítica, capacitado a atuar, visando à segurança alimentar e à atenção dietética, em todas as áreas do conhecimento em que a alimentação e nutrição se apresentem fundamentais para a promoção, manutenção e recuperação da saúde e para a prevenção de doenças de indivíduos ou grupos populacionais, contribuindo para a melhoria da qualidade de vida, pautado em princípios éticos, com reflexão sobre a realidade econômica, política, social e cultural (BRASIL, 2001b, p. 1). 
Dentre os nove cursos participantes deste estudo, apenas três (públicos) foram criados antes da LDB (especificamente na década de 1970). Os demais surgiram entre os anos de 2001 e 2011. Os cursos tem duração de oito a 10 semestres, com carga horária total entre 3.215 e 4.364 horas, sendo os mais antigos os que possuem maior duração e maior carga horária (o mínimo definido nas DCN é de 3.200 horas). Do grupo analisado, dois cursos são noturnos, ambos ofertados em instituições privadas.

Os dois cursos mais jovens (criados em 2006 e 2011) fazem parte de Universidades Federais, criadas no âmbito do processo de expansão e de interiorização do ensino superior público (BRASIL, 2007a). Cabe ressaltar que ambos foram implantados com uma estrutura curricular e pedagógica diferenciada dos demais participantes deste estudo, pois integram propostas de ensino superior público estruturadas sob os princípios da educação interprofissional e da integração ensino-serviço-comunidade.

Quanto à participação dos cursos nos programas de incentivo à reorientação da formação de profissionais de saúde para atender as demandas do SUS, seis participaram do Pró-Saúde (2 privados), e desses, quatro participaram também do PET-Saúde (1 privado).

As mudanças com vistas à qualificação da formação do nutricionista para o SUS tiveram sua implementação iniciada nesses cursos entre os anos de 2008 e 2015. Quatro cursos as realizaram no âmbito de um grande processo institucional de mudanças curriculares e inovações pedagógicas, que envolveu todos os cursos daquelas instituições ou pelo menos o conjunto dos cursos da área da saúde. As mudanças se relacionaram, especialmente, à promoção da integração ensino-serviço, à inserção oportuna e gradativa dos alunos na Rede de Atenção à Saúde e à assegurar uma abordagem integral do processo saúde-doença por meio da integração de conhecimentos e da articulação teoria-prática. Serão apresentados a seguir os aspectos mais inovadores e relevantes dessas mudanças, bem como os desafios enfrentados.

\section{Integração ensino-serviço}

Segundo Albuquerque et al. (2008, p. 357), a integração ensino-serviço é entendida como

[...] O trabalho coletivo, pactuado e integrado de estudantes e professores dos cursos de formação na área da saúde com trabalhadores que compõem as equipes dos serviços de saúde, incluindo-se os gestores, visando à qualidade de atenção à saúde individual e coletiva, à qualidade da formação profissional e ao desenvolvimento/satisfação dos trabalhadores dos serviços. 
Observou-se que a participação no Pró-Saúde e no PET-Saúde impulsionou a centralidade da integração ensino-serviço no processo de ensino-aprendizagem nas mudanças realizadas pelos cursos participantes desses Programas. No entanto, foi possível perceber melhor êxito nos cursos inseridos em cenários institucionais favoráveis para as mudanças empreendidas. Ou seja, quando não foi uma iniciativa específica do curso de Nutrição, mas em municípios onde foi construído/desenvolvido um projeto político-institucional de integração ensino-serviço, com participação de gestores e trabalhadores do SUS e das instituições de ensino (como Rede Docente Assistencial, Sistema de Saúde-Escola, Rede Escola, Política de Integração Ensino-Serviço).

Essa institucionalidade facilita a gestão, coordenada por processos administrativos e acadêmicos internos e suas interfaces externas, com potencial de reverter tendências que se apresentam hegemônicas na organização das práticas de ensino nos serviços de saúde, como: (a) a informalidade (falta de registro e controle); (b) a dispersão, a fragmentação e a descontinuidade (ações das IES espalhadas nos serviços sem nenhum critério nem ordenamento, não seguindo a lógica de organização do sistema de saúde, sem considerar as necessidades de saúde dos territórios); (c) a formação predominantemente no modelo biomédico (hospitalocêntrico, médico-centrado, tecnicista e curativista) e; (d) a priorização local e particular (facilidade de acesso para professores e alunos, relações pessoais dos docentes com servidores, disponibilidade pessoais dos servidores, dentre outros) (NEVES; AZZI, 2015; PIZZINATO et al., 2012).

Como ressaltam Brehmer e Ramos (2014), a integração entre os serviços de atenção à saúde e as IES exige, sobretudo, relações horizontais, processos de trabalho conjuntos, interesses em comum e alinhamento das necessidades e das potencialidades. Para tanto, é necessário que haja a promoção de espaços dialógicos entre as IES, as instituições de saúde e a comunidade. Nesse sentido, destacam-se, nas experiências estudadas que estavam inseridas em um projeto político-institucional de integração ensino-serviço, a existência de colegiado, de uma comissão permanente ou fórum para discussão, de planejamento e pactuação periódicos como, por exemplo, a cada início de semestre, a fim de delinear e avaliar a inserção dos estudantes de diferentes cursos e instituições na Rede de Atenção à Saúde local. Em algumas experiências, essa interlocução se estende para outros serviços dos territórios, como a Rede de Educação e a Rede de Assistência Social, contribuindo para uma formação mais ampla acerca do processo saúde-doença e do mundo do trabalho.

Sobre o ingresso do aluno no mundo do trabalho, Ferla (2015) destaca a importância da aproximação dos sistemas locais de saúde e demais políticas sociais que oferecem ações 
para as pessoas, de regras e organizações que gerem esses serviços, dos modos como esses impactam o território e se relacionam com as pessoas, e não mais do ingresso dos alunos apenas por meio de um ou outro serviço de saúde que não promova interface com a totalidade do sistema.

Nos cursos com abordagens metodológicas tradicionais, a busca pela inserção dos alunos nos serviços de saúde para além dos hospitais universitários, tende a ficar sob responsabilidade apenas dos docentes da área de Nutrição em Saúde Coletiva. Tal característica ocorre também devido às tendências hegemônicas na organização das práticas de ensino nos serviços de saúde, citadas anteriormente (NEVES; AZZI, 2015; PIZZINATO et al., 2012).

Os projetos de extensão, dentre eles o PET-Saúde, foram identificados por alguns cursos, como promotores de integração ensino-serviço-comunidade e como formas de oportunizar aos alunos uma melhor formação para a atuação no SUS. Importante destacar que os relatos de experiência apontam para a articulação das práticas extensionistas com estágios curriculares e atividades práticas de disciplinas, o que vai ao encontro à premissa de indissociabilidade entre ensino-pesquisa-extensão (SOUSA, 2005).

A concepção de extensão enquanto espaço real de ensino, articulando a teoria e a prática na graduação, é defendida por Sousa (SOUSA, 2005, p. 1) ao afirmar que

[...] a Extensão Universitária cria novos espaços na academia. A sala de aula deixa de ser o laboratório, a biblioteca, a sala convencional. Derrubam-se as paredes e destroem-se limites para as ações. Alunos e professores estão inseridos na realidade concreta experimentando o fazer acadêmico junto ao fazer profissional e tecendo relações sociais que refletem nas políticas públicas instituídas.

No quesito inserção dos alunos nos serviços de saúde, seis cursos (três públicos e três privados) conseguem realizá-la desde o primeiro semestre, com aproximações sucessivas, buscando o aumento progressivo da complexidade de reflexão/ação ao longo da formação. Cabe destacar que desses, cinco cursos estavam inseridos em municípios que possuíam um projeto político-institucional de integração ensino-serviço.

As atividades práticas nos serviços de saúde nos semestres iniciais dos cursos se referem, principalmente, ao reconhecimento e a compreensão dos determinantes sociais do processo saúde-doença e da organização dos serviços nos territórios. A inserção nos semestres mais avançados já é voltada para as práticas profissionais específicas do nutricionista. A partir dos relatos de experiência dos cursos, é possível atentar para o fato de que a inserção gradativa nos serviços de saúde contribui para que as práticas específicas dos graduandos em nutrição, como nos estágios curriculares finais, sejam mais contextualizadas e integradas às 
demandas da população e às ações dos profissionais atuantes nos serviços, o que se apresenta como potencialidade para a formação de profissionais com mais habilidades para a atuação em equipes multiprofissionais e na perspectiva da atenção integral à saúde (ALBUQUERQUE et al., 2008; CAVALHEIRO; GUIMARÃES, 2011).

As experiências nos serviços durante as aulas práticas e estágios contribuem para refletir sobre "[...] a prática do cuidado que ali é produzida e suas repercussões, inclusive sobre a maneira como se concebe o cuidado e se essa concepção se afasta ou se aproxima das manifestações presenciadas naquele espaço" (ALBUQUERQUE et al., 2008, p. 360). Mas, como ressaltam Albuquerque et al. (2008, p. 360), a vivência dos estudantes na realidade dos serviços de saúde "[...] não deve ser ponto de partida para críticas taxativas a estes serviços e seus profissionais, e sim funcionar como elemento instigador para uma prática problematizadora, no sentido da aprendizagem e também da reflexão sobre a produção dos cuidados".

Também, nesse sentido, Cavalheiro e Guimarães (2011, p. 26) destacam o necessário investimento em relações horizontalizadas, "[...] onde todo o produto e frutos são compartilhados, onde não há uma academia que simplesmente se utiliza do serviço como local de estágio, nem um serviço que se utiliza do estudante como mera mão de obra".

\section{Abordagem integrada do conhecimento}

Dos cursos que realizaram um grande processo institucional de mudanças curriculares e inovações pedagógicas, envolvendo todos os cursos da instituição ou os cursos da área da saúde, todos (sendo quatro privados) promoveram modificações por meio da integração de conhecimentos e da articulação teoria-prática. Ou seja, a abordagem integrada do conhecimento tem viabilizado, não apenas que a formação dos profissionais de saúde alcance uma formação cidadã, técnica e científica, como também os colocam em contato com a realidade de saúde da comunidade, possibilitando reflexões e elaborações para transformá-la. De forma adicional, os preparam para o trabalho em equipe e para a oferta de cuidado integral à saúde, compatíveis com o grau de autonomia (FEUERWERKE; SENA, 2004).

Nesse contexto, insere-se os pressupostos da interdisciplinaridade e a utilização de metodologias ativas de ensino e aprendizagem. Dentre as estratégias utilizadas nos cursos participantes, a abordagem integrada de conhecimento foi viabilizada por meio de eixos de formação, ciclos estruturantes ou por subunidades curriculares, sendo esses articulados por módulos distribuídos em núcleos temáticos interdisciplinares ou por conteúdos curriculares do 
curso articulados em torno de um objeto integrador. Observaram-se nas experiências temas relevantes e norteadores para a alimentação e nutrição como a segurança alimentar e nutricional, a alimentação adequada e saudável como direito humano básico, a ética, a bioética, o humanismo, dentre outros, perpassando integralmente todos os núcleos ao longo do curso.

Dentre as atividades que permitem viabilizar a integração do conhecimento nos cursos pesquisados foi possível observar a implementação de Atividades Integradoras ou de Integração (AI), Disciplinas Integradoras (DI), Projeto Integrador (PI), Fórum Integrador (FI) e/ou a interface com outros cursos de graduação ou Educação Interprofissional. Assim, nos ciclos ou núcleos onde era possível momentos de prática mais longos, foram inseridas algumas dessas atividades.

As AI propiciaram o uso da problematização como estratégia para viabilizar a integração do conhecimento, uma vez que conduzem o estudante a utilizar-se de todos os conhecimentos apresentados e adquiridos anteriormente, a partir de aproximações sucessivas das práticas, possibilitando a execução de tarefas de complexidade e de responsabilidade crescentes. Ou seja, essas atividades permeiam a combinação de conteúdos trabalhados teoricamente, com vistas à mobilização de recursos, elaboração de decisões e condutas frente a situações percebidas em contatos com a prática (MITRE et al., 2008; SOBRAL; CAMPOS, 2012). Essa estratégia pedagógica, baseada no Método do Arco, de Charles Maguerez, procura mobilizar o potencial político, social, e ético do sujeito para que esse atue "[...] como agentes sociais que participam da construção da história de seu tempo, mesmo que em pequena dimensão" (BERBEL, 1998, p. 33), enriquecendo a sua formação.

As dificuldades percebidas pelos cursos na sua implementação incluem a composição de grupos menores de alunos por turma, com o envolvimento de mais de um professor por AI, em cada semestre; a necessidade de uma atitude pró-ativa, de corresponsabilidade e de protagonismo por parte de todos os sujeitos envolvidos; além de implementação da cultura de uma educação permanente institucional. Destaca-se também a mudança de paradigma estrutural referente ao processo educativo, como o planejamento da carga horária e valorização do trabalho docente para elaborar, executar e avaliar as práticas implementadas contrapondo o modelo tradicional de formação e de produtividade. É exigida também uma intensa e constante articulação com a rede de serviços, bem como a presença de mais profissionais nutricionistas para que ocorra o vínculo ensino-serviço.

Para reforçar a formação, a realização de Fóruns de Atividades Integradoras viabilizou as escolhas dos temas a serem trabalhados semestralmente, o contato entre os atores em 
formação, o amadurecimento do processo de ajustes na formação, a articulação entre o ensino, a extensão e a pesquisa na IES, bem como com os programas de educação e de reorientação da formação profissional em saúde, por reforçarem na prática os objetivos dos mesmos. Nos cursos onde existiram esses Fóruns, observou-se mais inovação e melhores resultados.

Os dois cursos mais jovens, ambos de IES públicas direcionadas a cursos da área de saúde, estão inseridos em propostas diferenciadas no tocante à abordagem integrada do conhecimento. Uma delas possui sua organização curricular fundamentada na Aprendizagem Baseada em Problemas (Problem Based Learning - PBL), que objetiva tornar o estudante o agente ativo da sua aprendizagem, tendo o professor como facilitador do processo ensinoaprendizagem (MITRE et al., 2008; SOBRAL; CAMPOS, 2012). Nesse sentido, as aulas convencionais foram substituídas por sessões tutoriais, com práticas executadas em laboratórios, nos serviços de saúde ou outros espaços da comunidade, bem como atividades autodirigidas e sessões com participação de profissionais convidados, desde o início do curso.

Já a outra IES, adotou a Educação Interprofissional como um princípio norteador da estrutura curricular e do processo ensino-aprendizagem visando o desenvolvimento de competências para o trabalho em equipe na perspectiva da integralidade no cuidado em saúde. Esse tipo de abordagem prioriza que o estudante vivencie, em turmas mistas, o trabalho em equipe, a interdisciplinaridade e o compromisso com a integralidade das ações que deve ser alcançado com o reconhecimento e o respeito às especificidades de cada profissão (AGUILAR-DA-SILVA; SCAPIN; BATISTA, 2011; BATISTA; GERAB; BATISTA, 2013). Essa mudança de intencionalidade e de postura implica, não apenas no desenvolvimento de uma cultura de ensino-aprendizagem caracterizada pelas trocas e pelos saberes partilhados, estabelecendo espaços formativos mais significativos e mais comprometidos com a prática do trabalho em equipe, como também numa disposição para o diálogo e o planejamento integrado, o que requer novos formatos e formas de agir e de espaços nas IES (MARINS; REGO, 2011). Ou seja, exige um novo processo, que nem sempre é fácil de ser estabelecido, principalmente pela estrutura departamentalizada da maioria dos cursos superiores (VILELA; MENDES, 2003).

Apesar dos desafios, as abordagens do conhecimento, que se deram de maneira integrada, foram sinalizadas pelas IES como tendo contribuído no processo de formação do nutricionista para o SUS, ao apresentar momentos de interface entre teoria e prática precocemente no curso, favorecendo o momento de síntese do conhecimento nos estágios obrigatórios, promovendo a superação da fragmentação dos campos de conhecimento que compõem a formação desse profissional, mais investimento na capacitação dos docentes e no 
alcance da aquisição de habilidades para inovar. Observa-se que é possível propiciar o desenvolvimento de habilidades e competências, tornando os estudantes sujeitos mais ativos no processo ensino-aprendizagem e, portanto, com melhor formação para a atuação profissional. No entanto, esses resultados foram levantados apenas pelas IES que modificaram o aparelho formador no que se refere a perspectiva da integração. Em IES com metodologias mais tradicionais, onde a integração ocorreu apenas por meio de poucos momentos dentro do currículo, não foi possível observar tão expressivamente essas contribuições.

\section{Considerações finais}

Das experiências analisadas foi possível identificar que as estratégias indutoras de reestruturação da formação profissional, como o PET-Saúde e Pró-Saúde, contribuíram positivamente com os processos internos das IES e com o diálogo dessas com as secretarias, serviços e profissionais de saúde. Vale saudar que bons exemplos foram encontrados tanto em IES públicas quanto privadas.

Considerando que as mudanças necessárias ultrapassam os limites dos parâmetros técnicos e demandam uma nova cultura institucional, pode-se também observar que, as universidades e os cursos superiores mais jovens já iniciam as suas atividades com propostas de educação interprofissional e inserção precoce dos estudantes nos serviços. Essas IES também investem no diálogo qualificado com os serviços e profissionais da rede local de saúde, incluindo-os em etapas de planejamento pedagógico, e não apenas em momentos demandados por oportunidades de locais de prática, construindo um protagonismo conjunto e dialogado. Observa-se que cursos e universidades com metodologias tradicionais também têm conseguido avanços. Foram relatados desafios maiores no processo de articulação interna dos professores e instâncias superiores das IES e também no diálogo e no estabelecimento de parcerias externas que ampliem os formatos tradicionais de prática.

Coerente a esse aspecto, as reformulações que ocorreram no bojo de uma mudança mais ampla da IES, como por exemplo, na reforma ou na estruturação de todos os cursos de saúde, também alcançaram mudanças mais inovadoras, estruturais e sustentadas por ações complementares, como formação dos docentes, articulação bilateral entre instituições (universidade - secretaria de saúde) e não apenas pontuais (curso - serviço ou profissional).

As propostas curriculares que apostaram em processos de formação mais articuladas também investiram de maneira importante e sistemática na formação dos docentes, sejam em metodologias e práticas pedagógicas, sejam em atividades internas para a integração entre 
áreas de conhecimento e desenvolvimento de habilidades para a atuação de forma integrada com os serviços e profissionais. Esse, por sinal foi um aspecto levantado por todas as IES participantes do estudo. Naquelas onde há processos de formação permanente dos docentes, identifica-se seu papel estratégico e naquelas em que não há, evidencia-se a necessidade dessa perspectiva pedagógica de forma urgente.

Por fim, outro elemento que contribui para melhores e maiores resultados e sustentabilidade do PPP diz respeito aos sujeitos envolvidos no processo. Além do que já foi destacado sobre a inclusão dos profissionais de saúde envolvidos na gestão e nos serviços de saúde no planejamento do processo de formação, há também a necessidade de induzir a integração interna dos cursos. Aqueles que melhor se autoavaliaram têm promovido a articulação entre as áreas de formação do nutricionista, enfrentando o consenso de que esse tipo de iniciativa tem sido de responsabilidade quase que exclusivamente da área e dos docentes de nutrição em saúde coletiva. Se a grande meta é a formação profissional para o SUS e a atenção integral à saúde, os docentes de todas as áreas podem e devem se integrar aos processos de reformulação curricular. A continuidade desse estudo indica ser necessária a escuta de discentes e egressos, além de outros atores das IES, dos serviços de saúde e das comunidades envolvidos nesse processo, com vistas a superar a limitação observada no presente trabalho.

\section{Contribuidores e agência de fomento}

Esse estudo foi financiado por meio de uma Carta Acordo com a Organização Panamericana de Saúde (OPAS), com recursos da Coordenação Geral de Alimentação e Nutrição/Departamento de Atenção Básica/Secretaria de Atenção à Saúde/Ministério da Saúde.

\section{Referências}

AGUILAR-DA-SILVA, Rinaldo Henrique; SCAPIN, Luciana Teixeira; BATISTA, Nildo Alves. Avaliação da formação interprofissional no ensino superior em saúde: aspectos da colaboração e do trabalho em equipe. Avaliação, Campinas; Sorocaba, v. 16, n. 1, p. 167-184, 2011. Disponível em: < http://www.scielo.br/pdf/aval/v16n1/v16n1a09.pdf >. Acesso em: 03 out. 2016.

ALBUQUERQUE, Verônica Santos et al. A integração ensino-serviço no contexto dos processos de mudança na formação superior dos profissionais da saúde. Revista Brasileira de Educação Médica, Rio de Janeiro, v. 32, n. 3, p. 356-362, 2008. Disponível em: <http://www.scielo.br/pdf/rbem/v32n3/v32n3a10.pdf>. Acesso em: 15 ago. 2016. 
ALMEIDA, Márcio José. Diretrizes curriculares nacionais para os cursos universitários da área da saúde. Londrina: Rede Unida, 2003.

BATISTA, Sylvia Helena Souza Silva; GERAB, Irani Ferreira Silva; BATISTA, Nildo Alves. A interdisciplinaridade como princípio educativo em uma proposta de educação interprofissional em saúde: olhares docentes. ENCONTRO NACIONAL DE PESQUISA EM EDUCAÇÃO EM CIÊNCIAS, 9., 2013, Águas de Lindóia. Atas... Águas de Lindóia, nov. 2013. p. 1-8. Disponível em: <http://www.nutes.ufrj.br/abrapec/ixenpec/atas/resumos/R10271.pdf>. Acesso em: 3 out. 2016.

BERBEL, Neusi Aparecida Navas. A problematização e a aprendizagem baseada em problemas: diferentes termos ou diferentes caminhos? Interface - Comunicação, Saúde, Educação, Botucatu, v. 2, n. 2, 1998.

BERBEL, Neusi Aparecida Navas. As metodologias ativas e a promoção da autonomia de estudantes. Semina: Ciências Sociais e Humanas, Londrina, v. 32, n. 1, p. 25-40, 2011. Disponível em: < http://www.proiac.uff.br/sites/default/files/documentos/berbel_2011.pdf >. Acesso em: 03 out. 2016.

BRASIL. Institui diretrizes curriculares nacionais do curso de graduação em nutrição. Resolução CNE/CES no 5, de 7 de novembro de 2001. Diário Oficial da União, Brasília, Seção 1. p. 39, 2001a.

BRASIL. Resolução CNE/CES 5/2001. Institui Diretrizes Curriculares Nacionais do Curso de Graduação em Nutrição. Brasília: Ministério da Educação. Conselho Nacional de Educação. Câmara de Educação Superior, 2001b.

BRASIL. Resolução $n^{\circ} 330$, de 4 de novembro de 2003. Aplica 'os princípios e diretrizes para a norma operacional básica de recursos humanos para o SUS (NOB/RH-SUS)' como política nacional de gestão do trabalho e da educação em saúde, no âmbito do SUS. Diário Oficial da União, Brasília, 2003.

BRASIL. Portaria Interministerial MS/MEC No 2.101, de 3 de novembro de 2005. Institui o Programa Nacional de Reorientação da Formação Profissional em Saúde Pró-Saúde - para os cursos de graduação em Medicina, Enfermagem e Odontologia. Brasília: Ministério da Saúde. Ministério da Educação, 2005. Disponível em: < http://www.portaleducacao.com.br/odontologia/artigos/3454/portaria-interministerial-msmec-n-2101-de-3-de-novembro-de-2005 >. Acesso em: 03 out. 2016.

BRASIL. Decreto No 6096, de 24 de abril de 2007. Institui o Programa de Apoio a Planos de Reestruturação e Expansão das Universidades Federais - REUNI. Brasília: Presidência da República, Ministério da Educação, 2007a. Disponível em: <http://www.planalto.gov.br/ccivil_03/_Ato2007-2010/2007/Decreto/D6096.htm>.

BRASIL. Programa Nacional de Reorientação da Formação Profissional em Saúde Pró-Saúde: objetivos, implementação e desenvolvimento potencial. Brasília: Ministério da Saúde. Ministério da Educação, 2007b. Disponível em: < http://bvsms.saude.gov.br/bvs/publicacoes/07_0323_M.pdf >. Acesso em: 03 out. 2016.

BRASIL. Portaria Interministerial MS/MEC No 421 de 3 de março de 2010. Institui o Programa de Educação pelo Trabalho para a Saúde (PET Saúde) e dá outras providências. Brasília: Ministério da Saúde. Ministério da Educação, 2010. Disponível em: < http://bvsms.saude.gov.br/bvs/saudelegis/gm/2010/pri0421_03_03_2010.html >. Acesso em: 03 out. 2016. 
BRASIL. Política Nacional de Alimentação e Nutrição. Brasília: Ministério da Saúde. Secretaria de Atenção à Saúde. Departamento de Atenção Básica, 2012.

BRASIL. Cadastro Nacional dos Estabelecimentos de Saúde. TABNET CNES. Competência Maio/2016. Brasília: Ministério da Saúde. Departamento de Informática do SUS, 2016a. Disponível em: < http://cnes.datasus.gov.br/ >. Acesso em: 23 ago. 2016.

BRASIL. Lei $\mathbf{N}^{\circ}$ 9.394, de 20 de dezembro de 1996, que estabelece as diretrizes e bases da educação nacional. Atualizada até 8/6/2016. 12. ed. Brasília: Congresso Nacional. Câmara dos Deputados, 2016b. Acesso em: 23 abr. 2017.

BRASIL. Cadastro e-MEC de Instituições e Cursos de Educação Superior. Relatório Cursos de Nutrição. Brasília: Ministério da Educação, 2017. Disponível em: < http://emec.mec.gov.br/ >. Acesso em: 10 fev. 17.

BREHMER, L.C.F; RAMOS, F.R.S. Experiências de integração ensino-serviço no processo de formação profissional em saúde: revisão integrativa. Revista Eletrônica de Enfermagem, Goiânia, v. 16, n. 1, p. 228-237, 2014. Disponível em:

<https://www.fen.ufg.br/fen_revista/v16/n1/pdf/v16n1a26.pdf>. Acesso em: 4 ago. 2016.

CALADO, Carmem Lúcia Araújo. A expansão dos cursos de nutrição no Brasil e a nova Lei de Diretrizes e Bases da Educação. Brasília, 2003. Disponível em:

<http://www.cfn.org.br/novosite/pdf/expansao.pdf >.

CANESQUI, Ana; GARCIA, Rosa Wanda Diez. Antropologia e nutrição: um diálogo possível. Rio de Janeiro: Fiocruz, 2005.

CAVALHEIRO, Maria Teresa Pereira; GUIMARÃES, Alóide Ladeia. Formação para o SUS e os desafios da integração ensino serviço. Caderno FNEPAS, Rio de Janeiro, v. 1, p. 19-27, 2011. Disponível em:

<http://www.sbfa.org.br/fnepas/artigos_caderno/v11/artigo2_formacao_para_sus.pdf >. Acesso em: 03 out. 2016.

CECCIM, Ricardo Burg; FEURWERKER, Laura C. Macruz Mudança na graduação das profissões de saúde sob o eixo da integralidade. Caderno de Saúde Pública, Rio de Janiro, v. 20, n. 5, p. 1400-1410, 2004. Disponível em: 〈http://www.scielo.br/pdf/csp/v20n5/36.pdf〉. Acesso em: 3 out. 2016.

CONTERNO, Solange Fátima Reis; LOPES, Roseli Esquerdo. Pressupostos pedagógicos das atuais propostas de formação superior em saúde no Brasil: origens históricas e fundamentos teóricos. Avaliação, Campinas; Sorocaba, v. 21, p. 993-1016, 2016. Disponível em: $<$ http://www.scielo.br/scielo.php?script=sci_arttext\&pid=S1414$40772016000300993 \& \mathrm{nrm}=\mathrm{iso}>$. Acesso em: 03 dez. 2016.

FERLA, Alcindo Antônio Redes vivas de educação e saúde e a integração universidade e sistema local de saúde: saberes locais e múltiplas saúdes como capacidade profissional e como atributo das redes de atenção. In: FERLA, A. A. (Ed.). Redes vivas de educação e saúde: relatos e vivências da integração universidade e sistema de saúde. Porto Alegre: Rede UNIDA, 2015.

FEUERWERKE, Laura C. Macruz ; SENA, Roseni R. Brasil. Construção de novos modelos acadêmicos de Atenção à Saúde e de Participação Social. In: MINISTÉRIO DA SAÚDE (Ed.). Ver - SUS Brasil: cadernos de textos. Brasília: Ministério da Saúde, 2004. p.194-237.

GIL, Célia Regina Rodrigues et al. Práticas de interação ensino, serviços e comunidade: desafios e perspectivas de uma experiência de ensino-aprendizagem na atenção básica. Revista Brasileira de Educação Médica, Rio de Janiro, v. 32, n. 2, p. 230-239, 2008. 
Disponível em: <http://www.scielo.br/scielo.php?script=sci_arttext\&pid=S010055022008000200011\&lng=en\&nrm=iso>. Acesso em: 03 out. 2016.

MARINS, João José Neves; REGO, Sergio. Educação médica: gestão, cuidado, avaliação. São Paulo: Hucitec, 2011.

MEDEIROS, Maria Angélica Tavares de; BRAGA-CAMPOS, Florianita Coelho; MOREIRA, Maria Inês Badaró. A integralidade como eixo da formação em proposta interdisciplinar: estágios de Nutrição e Psicologia no campo da Saúde Coletiva. Revista de Nutrição, Campinas, v. 27, p. 785-798, 2014. Disponível em:

$<$ http://www.scielo.br/scielo.php?script=sci_arttext\&pid=S1415-

$52732014000600785 \& \mathrm{nrm}=\mathrm{iso}>$.

MENDES, Eugênio Vilaça As redes de atenção à saúde. Brasília: Organização PanAmericana da Saúde, 2011.

MITRE, Sandra Minardi et al. Metodologias ativas de ensino-aprendizagem na formação profissional em saúde: debates atuais. Ciência \& Saúde Coletiva, Rio de Janeiro, v. 13, n. Sup 2, p. 2133-2144, 2008. Disponível em: <http://www.scielo.br/pdf/csc/v13s2/v13s2a18.pdf >. Acesso em: 03 out. 2016.

NEVES, José Mário D'Ávila; AZZI, Lilia Maria Woitikoski. Caminhos da construção da Rede de Integração Ensino e Serviço da Secretaria Municipal de Saúde de Porto Alegre, RS. In: FERLA, A. A. (Ed.). Redes vivas de educação e saúde: relatos e vivências da integração universidade e sistema de saúde. Porto Alegre: Rede UNIDA, 2015.

PIZZINATO, Adolfo et al. A integração ensino-serviço como estratégia na formação profissional para o SUS. Revista Brasileira de Educação Médica, Brasília, v. 36, p. 170177, 2012. Disponível em: <http://www.scielo.br/scielo.php?script=sci_arttext\&pid=S010055022012000300025\&nrm=iso >. Acesso em: 03 out. 2016.

RECINE, E.; SUGAI, A. Consenso sobre habilidades e competências do nutricionista no âmbito da saúde coletiva. Brasília: Observatório de Políticas de Segurança Alimentar e Nutrição, 2013.

RECINE, Elisabetta et al. A formação em saúde pública nos cursos de graduação de nutrição no Brasil. Revista de Nutrição, Campinas, v. 25, n. 1, p. 21-33, 2012. Disponível em: < http://www.scielo.br/scielo.php?script=sci_arttext\&pid=S1415-

52732012000100003\&nrm=iso >. Acesso em: 03 out. 2016.

SOBRAL, Fernanda Ribeiro; CAMPOS, Claudinei José Gomes. Utilização de metodologia ativa no ensino e assistência de enfermagem na produção nacional: revisão integrativa.

Revista da Escola de Enfermagem da USP, São Paulo, v. 46, n. 1, p. 208-218, 2012.

Disponível em: < https://dx.doi.org/10.1590/S0080-62342012000100028 >. Acesso em: 03 out. 2016.

SOUSA, Ana Luiza Lima. Extensão universitária na UFG: Olhando para o passado. Revista da UFG, v. 7, n. 2, 2005. Disponível em: < http://www.proec.ufg.br/revista_ufg/45anos/Mextensao.html >. Acesso em: 03 out. 2016.

VILELA, Elaine Morelato; MENDES, Iranilde José Messias. Interdisciplinaridade e saúde: estudo bibliográfico. Revista Latinoamericana de Enfermagem, v. 11, n. 4, p. 525-531, 2003. 
${ }^{1}$ Elisabetta Recine Universidade de Brasília | Faculdade de Ciências da Saúde | Departamento de Nutrição Brasília | DF | Brasil. Contato: recine@unb.br ORCID (iD) 0000-0002-5953-7094

${ }^{2}$ Kelly Poliany de Souza Alves Universidade do Estado do Rio de Janeiro | Instituto de Medicina Social | Prog. de Pós-Grad. em Saúde Coletiva Rio de Janeiro | RJ | Brasil. Contato: kpsalves@gmail.com ORCID (iD) 0000-0001-7293-4628

${ }^{3}$ Estelamaris Monego Universidade Federal de Goiás | Faculdade de Nutrição Goiânia | GO | Brasil. Contato: estelamaris.monego@gmail.com ORCIID (iD) 0000-0002-3536-3165

${ }^{4}$ Andrea Sugai Universidade Federal de Goiás | Faculdade de Nutrição Goiânia | GO | Brasil. Contato: andreasugai @gmail.com

ORCIID (iD) 0000-0002-2185-9143

${ }^{5}$ Alice Cristina Medeiros Melo Organização Pan Americana da Saúde/ Organização Mundial da Saúde Brasília | DF | Brasil. Contato: acnmedeiros@gmail.com ORCID (iD) 0000-0003-1098-4747

Artigo recebido em 27 de outubro de 2017 e aprovado em 2 de abril de 2018. 